\title{
Quantum Information Science
}

\author{
M. Swan \\ Centre for Blockchain Studies, University College London \\ R. P. dos Santos \\ Science and Mathematics, Lutheran University of Brazil \\ F. Witte \\ Economics Department, University College London
}

\begin{abstract}
Quantum computing is implicated as a next-generation solution to supplement traditional von Neumann architectures in an era of post-Moore's law computing. As classical computational infrastructure becomes more limited, quantum platforms offer expandability in terms of scale, energy-consumption, and native three-dimensional problem modeling. Quantum information science is a multidisciplinary field drawing from physics, mathematics, computer science, and photonics. Quantum systems are expressed with the properties of superposition and entanglement, evolved indirectly with operators (ladder operators, master equations, neural operators, and quantum walks), and transmitted (via quantum teleportation) with entanglement generation, operator size manipulation, and error correction protocols. This paper discusses emerging applications in quantum cryptography, quantum machine learning, quantum finance, quantum neuroscience, quantum networks, and quantum error correction.
\end{abstract}

\section{INTRODUCTION}

Quantum computing is the use of engineered quantum systems to perform computation, meaning physical systems comprised of quantum objects (atoms, ions, photons) manipulated through configurations of logic gates. "Quantum" refers to the scale of atoms (nanometers $10^{-9}$ ), ions and photons (picometers $10^{-12}$ ), and subatomic particles (femtometers $10^{-15}$ ). The general expectation in quantum computing is a quadratic speedup in the computation of certain kinds of equations. Algorithms are being rewritten to take advantage of the quantum speedup in processing linear algebra routines, Fourier transforms, and other optimization tasks.

The development of quantum technologies is a worldwide effort spanning several hardware platforms that are currently available via cloud services (e.g. IBM Q 27-qubit, IonQ 32-qubit, Rigetti 19Q Acorn systems) and that have been announced in research efforts
(Google's 53-qubit Sycamore quantum processor) Contemporary quantum systems are non-error corrected NISQ (noisy intermediate-scale quantum) devices constrained to 50-100 qubits. The million-qubit systems needed for full-scale quantum operations (such as Shor's factoring algorithm and Grover's search algorithm) are not immediately immanent. Technical breakthroughs are needed to deliver the quantum error correction required to progress from NISQ devices to fully FTQC (fault-tolerant quantum computing) [1]. However, progress in many areas of foundational physics is enabling new technologies that translate to practical use in quantum computing, for example a black-hole-on-a-chip formulation and experimental setups for testing quantum gravity in the laboratory [2].

\section{Superposition and Entanglement}

Superposition and entanglement are two counterintuitive features that are harnessed in quantum

IEEE Internet Computing $\quad$ Published by the IEEE Computer Society $\quad$ XXXX-XXXX@ 2022 IEEE 
computation. Superposition is the quantum property of an unobserved particle existing in all possible states simultaneously, until collapsing to only one when measured. Entanglement is the quantum property of physical attributes being correlated between particles (position, momentum, polarization, spin), even when separated by distance (if one particle is in a spin-up state, the other is in a spin-down state; a "heads-tails" relationship). The scalability advantage of quantum computing is seen in the superposition-based modeling of data as the quantum information representation of all possible system states simultaneously.

\section{QUANTUM CRYPTOGRAPHY}

One of the most immediate high-profile applications of quantum computing is cryptography. The "Y2K of crypto" problem is that practical quantum computing will break the security of nearly all modern public-key cryptographic systems (based on SHA-256 and related hashing algorithms) [3]. Post-quantum cryptographic algorithms that are resistant to quantum computerbased attacks will need to be implemented in a worldwide roll-out effort. The development of quantum-resistant public-key cryptographic standards is underway with the algorithm selection process expected to be completed by 2023. In June 2021, the U.S. NIST announced that their Post-Quantum Cryptography Standardization Process had entered the third phase, with 7 third-round finalists and 8 alternate candidates being considered for standardization. The mainstay application for post-quantum cryptography is quantum key distribution on global networks.

Quantum-secure algorithms mainly involve a shift to mathematics based on lattices (group theory) as opposed to factoring (number theory). Some of the first quantum algorithms developed to take advantage of non-classical properties are Shor's quantum factoring algorithm and Grover's quantum search algorithm. Shor's algorithm is a period-finding function with a quantum Fourier transform (a classical discrete Fourier transform applied to the vector amplitudes of a quantum state), which is exponentially faster than classical algorithms (the general number field sieve). More recently proposed quantum-secure cryptographic methods include access based on location instead of authorization, with quantum secret sharing localized to spacetime, and authorized regions replacing authorized parties. Time entanglement (entanglement in time instead of space) is also possible for cryptographic key exchange within a short time window.

\section{Entropic Uncertainty}

The potential advent of global quantum networks, with end-to-end qubit delivery and entanglement as a service, suggests that quantum properties such as entropy might be engaged for a sophisticated slate of applications [4]. Entropy has a specific meaning in the second law of thermodynamics in that the total entropy of an isolated system cannot decrease over time. More generally, entropy quantifies the number of microscopic configurations (microstates) that are consistent with the macroscopic properties of a thermodynamic system (volume, pressure, temperature). Entropy is central to information science as the minimum number of bits (qubits) needed to communicate a message, and for measuring the interrelatedness of subsystems within a system.

Entropic uncertainty is a stronger more-computable version of the Heisenberg uncertainty principle that concretizes the trade-off between conjugate variables such as position-momentum and energy-time (frequency); the more that is known about a particle's position, the less that can be known about its momentum. Entropic uncertainty constitutes the lower bound of the Heisenberg uncertainty principle and is straightforward to calculate as min-entropy (using entropy instead of standard deviation).

Min-entropy (minimum entropy) measures the amount of uncertainty (entropy) in a system by quantifying the degree of uniformity in the distribution of a random variable (as a lower bound of the sum of entropies comprised by the temporal and spectral Shannon entropies or (equivalently) as the quantum generalization of conditional Rényi entropies). The interpretation is that the lower the min-entropy, the higher the certainty of the system producing a certain outcome (unbreakable cryptography for example). Quantum systems can also be upper-bounded using Holevo's theorem to restrict the amount of information known about a quantum state.

\section{QUANTUM MACHINE LEARNING}

Quantum machine learning (machine learning applied in a quantum environment) is emerging as one of the first potential general-purpose applications of near-term quantum devices. A key advance is the Born machine as the quantum version of the Boltzmann machine [5]. Both "machines" are automated energy functions that evaluate probability output from machine learning algorithms. Classically, the Boltzmann machine uses an energy-minimizing 
probability function for sampling (per the Boltzmann distribution in statistical mechanics). The Born machine interprets results with the Born rule (a computable quantum mechanical formulation that evaluates the probability density of finding a particle at a given point as being proportional to the square of the magnitude of the particle's wavefunction at that point).

\section{Born Machine and QGANs}

Born machines facilitate a substantial shift into unsupervised learning (the classification of unlabeled data). Whereas in supervised learning, discriminative networks learn from already-labeled data, in unsupervised learning, generative networks learn from the distribution of data such that they can create new samples. Generative adversarial networks (GANs) are a machine learning technique that pits two networks (discriminator and generator) against each other: the generator produces new samples, and the discriminator distinguishes between real and false samples.

The discriminator provides a gradient (slope) which the generator uses for gradient-based learning. The gradient is a cost function that optimizes the weights of different model variables with respect to their error contribution. A further advance is QGANs (quantum GANs), which use quantum circuits to construct the generative adversarial network and to compute quantum gradients. Quantum gradients are the quantum version of gradients derived using variational quantum eigensolvers (VQEs), a standard quantum algorithm.

\section{Reproducing Kernel Hilbert spaces (RKHS)}

The main approaches to machine learning are neural networks, tensor networks, and kernel learning, each of which has a quantum counterpart. Quantum kernel learning methods are particularly conducive to the multiscalar environments of quantum systems [6]. For example, the RKHS (reproducing kernel Hilbert space) formalism is used to compute kernel functions at various scales of a quantum system. This enables a more comprehensive form of pattern recognition which is of interest in quantum finance.

\section{Pixel $=$ Spin (Qubit)}

Tensor networks are likewise conducive to quantum machine learning as they also treat multiscalar quantum systems [7]. Tensor networks are a tool for efficiently representing and solving quantum states (highdimensional data in the form of tensors). Tensor networks operate by factoring a high-order tensor (a tensor with a large number of indices) into a set of loworder tensors whose indices can be summed (contracted) in the form of a network, and are used to renormalize entanglement across scale tiers. A key tensor network insight for quantum machine learning is that each pixel can be modeled as a spin (qubit) for point data ("pixel = qubit"), and similarly, each wavelet (wave element) can be modeled as a spin for sequential data ("wavelet = spin"). The entanglement renormalization feature of tensor networks can be used in quantum machine learning, for example, to identify short-range correlations as being most relevant to a standard (MNIST) image classification task [8].

\section{Fourier Neural Operators}

Neural networks too are being further extended into the quantum domain with Fourier neural operators [9]. Operators (mathematical functions) are used to manipulate quantum systems indirectly since acting directly would result in wavefunction collapse before the calculation is complete. Partial differential equations (PDEs) are often applied to solve for the multiple unknown variables in wavefunctions (as opposed to ordinary differential equations (ODEs) which solve for just one unknown variable).

Two traditional methods for solving PDEs are conventional solvers and data-driven solvers. Conventional solvers proceed by discretizing the problem space and time into grids, either with coarse grids that are fast but imprecise or fine grids that are accurate but slow. Data-driven solvers offer a faster automated method with machine learning. However, the solution is still classically limited as it can only map between finite-dimensional spaces and learn solutions related to one specific discretization at a time.

Neural operators provide an advance for the quantum domain as a neural network architecture that is able to map between infinite-dimensional spaces. The operator is a complex mathematical function that has the flexibility to address infinite-dimensional spaces within a single set of network parameters. To solve ODEs, neural operators can be defined in the form of a neural network whose weights are smooth functions of continuous depth, in which input is evolved to output with a trainable differential equation (as opposed to mapping between discrete layers).

To solve PDEs, a requirement is two dimensions of multiplicity, both the infinite spaces within which the functions operate, and the many permutations of variables related to the instances of the PDE family. The first neural PDE methods solved either all instances of the PDE family in one spatial discretization, or one instance of the PDE family in 
multiple spatial discretizations. A further advance is the Fourier neural operator, a quantum deep learning architecture that learns mappings between the infinitedimensional spaces of functions and solves for the entire PDE family (by parameterizing the integral kernel directly in Fourier space).

\section{Quantum Information Scrambling}

An advanced quantum machine learning application is using quantum information scrambling and entropic uncertainty as methods for efficient Hilbert space exploration in quantum circuit design for machine learning and benchmarking [10]. Quantum information scrambling is a measure of how quickly information spreads out in a quantum system so that a local measurement is no longer possible. The information is not lost and may be recovered later in a different part of the system (which has implications for quantum teleportation, memory, and cryptography). Quantum information scrambling has been demonstrated in Google's 53-qubit system.

\section{QUANTUM FINANCE}

Quantum finance is the application of physics methods to problems in finance, namely, option pricing, trading strategies, risk management, and portfolio optimization. Markets have long been modeled as complex physical phenomena per the principles of wavefunctions, thermality, dissipation, and Brownian motion, and now these models are being implemented with real-life quantum hardware instead of simulation. Quantum finance could be one of the first mainstream fields to develop in quantum computing as the financial industry is typically an early adopter of new technologies, and currently finds itself in a potential progression from classical to digital (blockchains) to quantum methods.

So far, the main quantum algorithms deployed in finance include quantum amplitude estimation algorithms, quantum Monte Carlo methods, anharmonic oscillators, and quantum kernel learning. Quantum amplitude estimation algorithms are used to estimate the properties of random distributions such as risk measures [11]. A quantum circuit is defined with a unitary operator to act on a register of qubits and an estimation operator to operate on the system based on quantum phase estimation (approximating certain eigenvalues of the estimation operator). Qubits are put into superposition (by applying Hadamard gates) and used to control different powers of the estimation operator. The system is evolved (by applying an inverse quantum Fourier transform) and the qubit state is measured. The result is an integer which is mapped to a classical estimation function. Streamlining the phase estimation process requires even fewer gates. Tested on the IBM Q 20-qubit Tokyo system, the method indicates a quadratic speedup compared to the convergence rate of classical Monte Carlo methods.

Other quantum finance projects use quantum amplitude estimation likewise for bond portfolio valueat-risk assessment per interest rate movement, anharmonic oscillators to define a price-energy relationship in Schrödinger wavefunctions for asset pricing and market dynamics, a quantum circuit Born machine to analyze S\&P500 time-series data for portfolio optimization, and a quantum kernel learning method based on reproducing kernel Hilbert spaces as a data classification method for trade selection.

\section{Quantum Walks}

Quantum walks are the quantum version of the random walk used in finance to characterize market behavior on the model of Brownian motion [12]. Classical random walks are based on Markov (stochastic) processes and a coin flip, and quantum walks too proceed on the basis of a coin flip, using a quantum coin-flip operator such as a Hadamard coin (which flips a qubit into a one or zero). Quantum walks also have more variables to select including the multidimensional graph structure of the walk environment, the walk algorithm, the time regime (discretecontinuous), and the coin operator.

Whereas random walks occur in the flat spatial structure of a two-dimensional grid, quantum walks proceed in the multi-dimensional space of a Bloch sphere, per the mathematics of lattice graph structures. The lattice graphs might be further embedded in a Euclidean plane to form regular tessellation patterns (triangular, square, and honeycomb lattice graphs).

Quantum walks are faster than random walks as they have ballistic propagation through a lattice graph as opposed to classical algorithms which are constrained to diffusive spread. Another factor is interference effects which allow the quantum walk to penetrate decision-tree structures more quickly. This enables the primary application of quantum walks which is finding faster algorithms, for example in cryptography and search. The faster the algorithm, the safer it is cryptographically (a hacker has less time to infiltrate 
the system) and the faster the search results are delivered (e.g. to identify a trading opportunity).

\section{Quantum Blockchains}

Quantum blockchains are proposals for blockchains (distributed ledger systems) that use quantum methods for post-quantum cryptography and the underlying blockchain protocols. As cryptographic systems, blockchains are acutely exposed to the risk of practical quantum computing, and would be likely to upgrade quickly once post-quantum cryptographic algorithms become standard. Quantum key distribution is an important first-tier application for quantum blockchains, and also continuous-time quantum walks which are faster (and therefore more secure) than classical random walks. Blockchain protocols might be formulated with optical networks that rely on entangled GHZ (Greenberger-Horne-Zeilinger) states, and use anharmonic oscillators for computational verification (zero-knowledge proofs).

\section{QUANTUM NEUROSCIENCE}

Quantum neuroscience is the application of quantum information science methods to problems in neuroscience, particularly wave-based analysis, quantum biology state modeling, and neuroscience physics [13]. Some of the first quantum neuroscience applications use quantum machine learning to study EEG data with quantum neural networks, for example proposing a quantum circuit to extract 794 features from 21 EEG channels to evaluate Parkinson's disease patients for deep brain stimulation treatment. One research project has developed quantum algorithms to reconstruct medical images from MRI, CT, and PET scans (MRI images with an inverse Fourier transform, and CT and PET images with an inverse Radon transform and the Fourier slice theorem). Another project integrates EEG and fMRI wave-based data at various spatiotemporal scales and dynamics regimes. A key finding is that whereas epileptic seizure can be explained by chaotic neural dynamics, normal resting states are more complicated, and are perhaps explained by bifurcation neural dynamics, in which there is an orbit-based organizing parameter periodically interrupted by countersignals to trigger a neural signal.

The second level of quantum neuroscience applications is quantum biology state modeling, which involves superpositioned data (the superposition-based modeling of data as the quantum information representation of all possible system states simultaneously) and quantum probability [14].
Biological systems constantly interact with their environment and change their behavior as a result. Models thus include updating processes in which the system takes measurements and responds accordingly.

Quantum biological states are interpreted with quantum probability, quantum mechanical rules for assigning probabilities which accommodate interference effects that violate the law of total probability, and commutativity in conjunction, in classical systems. A quantum variant of total probability can be obtained with an interference term for incompatible observables given by POVMs (positive operator valued measures), positive measures on a quantum subsystem of the effect of a measurement performed on the larger system. The main interpretation of quantum probability is with the Born rule, but there can be others. Quantum Bayesian methods, notably QBism ("cubism"), incorporate subjective (observer-based) aspects.

The third level of quantum neuroscience applications is neuroscience physics (the neuroscience interpretation of foundational physics findings). Using operators to evolve the system is a key requirement for the study of quantum systems. Traditionally, quantum system evolution involves Schrödinger and Heisenberg dynamics. However, the Heisenberg equation of motion is only a general approximation of movement and does not include temperature, and the Schrödinger wavefunction is limited to describing pure quantum states as opposed to mixed (subsystem) states. Contemporary methods for quantum system evolution thus include ladder operators and master equations.

Ladder operators (creation-annihilation operators) are a standard quantum operator used to raise and lower quantum system tiers to evolve systems (by acting to increase or decrease the eigenvalue of another operator). Ladder operators are a first-line approach, and more detailed quantum system evolution is provided by master equations. The quantum master equation is the quantum version of the classical master equation (describing the time evolution of a system as a probabilistic combination of states); the simplest form is the Lindblad equation (Lindbladian), a quantum Markov model (stochastics with quantum probability).

The AdS/Brain theory is introduced as the first multi-tier interpretation of the AdS/CFT correspondence [13]. The AdS/CFT (Anti-de Sitter Space/Conformal Field Theory) correspondence is a theory that any physical system with a bulk volume can be described by a boundary theory in one less 
dimension. The AdS/Brain theory is a composite model of neural signaling in the form of a multi-tier renormalized bMERA tensor network (brain multiscale entanglement renormalization ansatz). The model involves four successive bulk-boundary pairs at the scale tiers of brain network, neuron, synapse, and molecule, all of which are involved in neural signaling.

Other neuroscience physics projects propose AdS/Memory (an information storage model based on excited state energy-tiers), a neuronal gauge theory (in which the system's global symmetry parameter (free energy) is rebalanced by gauge fields after signaling), and a superconducting ordered-disordered phase transition model of neural signaling.

\section{QUANTUM NETWORKS}

Global-scale quantum networks are envisioned for ultra-fast, ultra-secure communication, computation, and sensing. Such networks would likely be photonic, relying on expertise in global fiberoptic communications dating from the 1960s. A full-stack roadmap (analogous to the OSI stack) is proposed for end-to-end qubit delivery and heralded entanglement [4]. Entanglement generation is a central requirement for quantum networks (for quantum key distribution and quantum teleportation), and various plans outline distilled, swapped, heralded (confirmed), and highdimensional entanglement.

\section{Qubits and Qudits}

The basic unit of quantum information is the qubit (quantum information bit), a two-state quantum mechanical system used for computing, as the quantum version of the classical bit. However, qubits are just one example of the broader class of qudits (quantum information digits) since a unit of quantum information can exist in any number of states. A qubit is a binary quantum information bit taking the values of 0 and 1 , and exists in a superposition of 0 and 1 before measurement. A qutrit is a trinary quantum information bit taking the values of 0,1 , and 2 , and exists in the 0 , 1 , and 2 states until measured.

Qudit-based systems offer greater efficiency than binary systems, for example, the qutrit as a three-state unit configures a self-contained nine-state errorcorrection code system. Qudits are routinely produced and transmitted in photonic networks, including with high-dimensional quantum entanglement (entangling particles in more than one dimension simultaneously, such as polarization, spatial modes, propagation path, and time-frequency bins).

\section{Quantum Teleportation}

Quantum teleportation is the process of sending a quantum state (the information state of a quantum system) from one location to another, relying on entangled particle pairs (Bell pairs). Transmission protocols measure the UV-IR correlations in a system, how entangled subsystems are within a system at shortrange (ultraviolet (UV)) and long-range (infrared (IR)) scales. Video streaming protocols (in the classical domain) use UV-IR correlations as movies have little frame-to-frame change during scenes (short-range correlation), and longer-range correlation based on the fact that the faces of the main characters do not change, but their expressions do. The same mathematics apply in defining quantum teleportation protocols based on the UV-IR correlations of the system.

As operators orchestrate the quantum computation process, they are likewise central to quantum teleportation [2]. Transmission protocols are defined on the basis of operator parameters. For example, winding and unwinding operator size distributions are a means of guessing wavefunctions relevant to transmission. Whereas conventional-size distributions uniformly sum amplitude coefficients to approximate the wavefunction, winding-size distributions use a method in which coefficients in the size basis acquire an imaginary phase that accelerates the winding and unwinding of operator size distribution. The result is teleportation-by-operator-size, a more efficient information compression protocol. Specific to manybody quantum teleportation, operator growth spreading and peaked-size teleportation protocols are defined.

An advanced method used in quantum teleportation is out-of-time-order correlation functions (OTOCs) [15]. OTOCs are used to evolve a quantum system backward or forward in time to apply actions and measure the system. The computation involves calculating the rate of system divergence by comparing how fast two initially-commuting operators decay to become non-commuting. OTOCs indicate whether a quantum system is chaotic and its scrambling time.

Chaos describes the evolution of many classical and quantum systems, indicating that seemingly random states are in fact governed by deterministic laws and sensitivity to initial conditions. Quantum chaotic systems are characterized by growth eras of initially 
ballistic growth that then slows and saturates, as described by the Lyapunov exponent (a quantification of the butterfly effect). Complex systems are generally found to be chaotic and fast-scrambling.

\section{口 QUANTUM ERROR CORRECTION}

The central factor in the long-term feasibility of quantum computing is error correction [1]. Error correction protects quantum information states from environmental noise. Noise arises in communications networks as quantum states pass through noisy channels, and in quantum computing as additional error classes are introduced in information processing.

Classical error correction methods such as making redundant copies or checking information integrity before transmission are not possible in quantum systems since information cannot be copied or inspected (per the no-cloning and no-measurement principles of quantum mechanics). Quantum error correction therefore often relies on entanglement instead of redundancy. The quantum state to be protected is entangled with a larger group of states from which it can be corrected indirectly (one qubit might be entangled with a nine-qubit ancilla of extra qubits).

The standard errors are a bit flip, a sign flip (the sign of the phase), or both. Basic codes diagnose the error, corresponding to Pauli matrices for controlling qubits in the $\mathrm{X}, \mathrm{Y}$, and $\mathrm{Z}$ dimensions. The error is expressed as a superposition of basis operations given by the Pauli matrices. If there is an error, the same Pauli operator is applied to act again on the corrupt qubit to reverse the error effect. The unitary correction returns the state to the initial state without measuring the qubit directly.

The basic form of the quantum error-correcting code is the stabilizer code (applied via ancilla), as the quantum version of linear codes used classically. In quantum computing, topology-based stabilizer codes (the toric code and the surface code) are proposed as a potential method for fault-tolerant error correction. Particle movement and its correction are interpreted in the structure of lattice topologies. Toric codes are stabilizer codes defined on a two-dimensional lattice with periodic boundary conditions (thus giving the shape of a torus), with stabilizer operators on the spins around each vertex and plaquette (face). Surface codes are a more generic formulation of topology-based stabilizer codes, which are also defined on twodimensional spin lattices, and take various shapes, but are not necessarily toroidal. Lattice surgery is a method of switching between codes on the fly.
There are two kinds of quantum gates, Clifford gates and non-Clifford gates (based on Clifford algebras). Clifford gates are basic quantum gates (Pauli matrices, and the Hadamard, CNOT, and $\pi / 2$-phase shift gates) that can be simulated efficiently on a classical computer (i.e. in polynomial time). Non-Clifford gates are needed for greater logical depth and more complex operations (such as the $\pi / 8$ gate), and cannot be simulated efficiently on a classical computer.

Magic state distillation is used to implement nonClifford gates, a computationally-expensive task of consolidating multiple noisy quantum states into a few reliable states. A more efficient alternative is gauge fixing. One proposed code based on gauge fixing is a stabilizer code related to Majorana fermion braiding and color codes (gauge color fixing). Another is a timebased surface code that replicates the threedimensional code that performs the non-Clifford gate functions with three overlapping copies of the surface code interacting locally over a period of time.

\section{CONCLUSION}

Quantum information science is a field with substantial progress and near-term opportunity, but also risk. It may be too early in the technology cycle if faulttolerant error correction methods cannot be found and platforms do not progress beyond hundred-qubit systems. Sharper critiques are that more foundational quantum algorithms should have been discovered by now, that quantum problem-solving may be limited to certain kinds of operations (e.g. linear algebra), and that a full-blown quantum infrastructure (processors, repeaters, networks, memory) is needed to assess the potential contribution of the technology.

However, despite these challenges, it is not too early to reformulate classical problems for computing in the quantum domain, and begin testing on quantum simulators and near-term quantum hardware available via cloud services. At minimum, quantum computing provides an alternative platform to ease the burden on classical von Neumann architectures in a moment of transitioning to post-Moore's law computational solutions. Overall, quantum computing emerges as a novel computational platform which provides a more capacious architecture with greater scalability and energy-efficiency than current methods of classical computing and supercomputing, and more naturally corresponds to the three-dimensional structure of atomic reality. 


\section{Quantum Information Science}

\section{REFERENCES}

1. J. Preskill, "Quantum computing 40 years later," 2021, arXiv: 2106.10522.

2. A. R. Brown et al., "Quantum Gravity in the Lab: Teleportation by Size and Traversable Wormholes," 2019, aXiv:1911.06314v1.

3. W. Barker, M. Souppaya, and W. Newhouse, "Migration to Post-Quantum Cryptography," NIST National Institute of Standards and Technology and National Cybersecurity Center of Excellence, pp. 1-15, 2021.

4. A. Dahlberg et al., "A Link Layer Protocol for Quantum Networks," Proc ACM SIGCOMM 2019, pp. 1159-73, 2019.

5. S. Cheng, J. Chen, and L. Wang, "Information perspective to probabilistic modeling: Boltzmann machines versus Born machines," Entropy, vol. 20, no. 583, 2018.

6. M. Schuld and N. Killoran, "Quantum machine learning in feature Hilbert spaces,” Phys. Rev. Lett., vol. 122, no. 040504, 2019.

7. E. M. Stoudenmire and D. J. Schwab, "Supervised learning with quantum inspired tensor networks," Adv. Neural Info. Proc. Sys., vol. 29, no. 4799, 2017.

8. J. Martyn, G. Vidal, C., Roberts, and S. Leichenauer, "Entanglement and tensor networks for supervised image classification," 2020, arXiv: 2007.06082v1.

9. Z. Li et al., "Fourier neural operator for parametric partial differential equations," 2021, arXiv:2010.08895v3.

10.X. Mi et al., "Information Scrambling in Computationally Complex Quantum Circuits," 2021, arXiv:2101.08870v1.

11.N. Stamatopoulos et al., "Option pricing using quantum computers," Quantum, vol. 4, no. 291, 2020.

12. V. Kendon, "How to Compute using Quantum Walks," EPTCS, vol. 315, 2020.

13. M. Swan, R. P. dos Santos, M. A. Lebedev, and F. Witte, Quantum Computing for the Brain, London: World Scientific, 2022.

14. I. Basieva, A. Khrennikov, and M. Ozawa, "Quantum-like modeling in biology with open quantum systems and instruments," BioSystems, vol. 201, no. 104328, 2021.

15. B. Swingle, G. Bentsen, M. Schleier-Smith, and P. Hayden, "Measuring the scrambling of quantum information," Phys. Rev. A., vol. 94, no. 040302, 2016.

Melanie Swan is a Research Associate at the Centre for Blockchain Technologies at University College London with research interests in emerging science and technology, particularly as directed towards biology, with projects in genomics, anti-aging, and brain-computer interfaces. Contact: melanie@blockchainstudies.org.

Renato P. dos Santos is a Researcher in blockchain technologies and a Graduate Professor at the Lutheran University of Brazil. His research interests include cryptocurrencies, data science in STEM education, Web 2.0 technologies, ethnoscience, physics teaching, artificial intelligence and computer algebra in physics, and quantum field theory. Contact: renatopsantos@ulbra.edu.br.

Frank Witte is an Associate Professor of Economics at University College London. His research focusses on the applications of physics-inspired methods to problems in Environmental Economics, Economics of Science, and the Economics of Networks. Contact: f.witte@ucl.ac.uk. 